\title{
EL FUTURO DEL PASADO. UNA PERSPECTIVA CRÍTICA DESDE LA UNIVERSIDAD DE SALAMANCA
}

\author{
The Future of the Past. A critical approach from the University of Salamanca perspective.
}

\author{
Iván PÉREZ MIRANDA* \\ Universidad de Salamanca \\ E-mail: ivan@usal.es
}

Fecha de recepción: 10-01-2010

Fecha de aceptación: 29-01-2010

\begin{abstract}
RESUMEN: Este artículo se plantea como reflexión general, desde la perspectiva de la universidad de Salamanca, sobre los mecanismos de poder que condicionan la situación de la investigación histórica, la divulgación, la crítica y la promoción académica en España.

Palabras clave: investigación, divulgación científica, crítica, calidad de las publicaciones, promoción académica.
\end{abstract}

ABSTRACT: This paper is presented as a general consideration about the mechanisms of power that determine the situation of the historical research, the publications, the critique, and the academic promotion in Spain, from a University of Salamanca perspective.

Keywords: Research, Scientific spreading, Critique, Quality of publications, Academic promotion. 
Incluso el pasado puede modificarse; los historiadores no paran de demostrarlo. Jean Paul Sartre.

En 1927, un físico alemán, Werner Heisenberg, formuló el principio de incertidumbre, también conocido como la relación de indeterminación de Heisenberg${ }^{1}$. Según este principio, el observador, por el mero hecho de estudiar la realidad, la altera, introduce una variable de indeterminación. Este principio se aplica en física cuántica pues, cuando el investigador mide la posición y velocidad de un electrón, las altera haciendo que un fotón de luz choque contra él, no siendo posible la atención al espacio y al tiempo como algo alejado del sujeto, que pasa a formar parte del sistema. Puede decirse entonces que ningún sistema puede ya ser definido como algo objetivo, separado del investigador que lo estudia, sino sólo en su relación compleja y dinámica con ese mismo sujeto².

Si aplicamos estas sugerentes ideas a la Historia, podemos afirmar que el historiador, al estudiar el pasado, lo cambia, le da forma, lo construye, pues el pasado, por definición, ya no existe. El historiador construye el pasado en y desde el presente. Nunca se enfrenta al pasado para contemplar lo que éste le ofrece, sino que selecciona, desde el presente, el tema y los problemas que le interesan ${ }^{3}$. La realidad que transmite el discurso histórico es una realidad mediada, y el historiador debe ser consciente de que no existe una verdad absoluta e invariable (más allá de la fe), sino que esta siempre es histórica, mutable en el tiempo. Siguiendo a Domingo Plácido:

La percepción del cambio debe tener un punto de referencia en uno mismo, que también se halla en constante cambio. Por ello, el historiador es un constante analista de sí mismo y de sus transformaciones en relación con el mundo exterior. La conciencia de la subjetividad y de sus relaciones con la totalidad abre las puertas para la percepción de la totalidad histórica, como inalcanzable, pero que se impone como instrumento en la comprensión del mundo exterior. 
Ahora bien, como ponía de manifiesto Juan Cascajero:

afirmar la presencia de la subjetividad no implica lesión alguna a la deseada objetividad. Porque, en el discurso temporal de toda reflexión histórica, subjetividad y objetividad se refuerzan recíprocamente en un continuo e inexorable proceso de enriquecimiento dialéctico 5 .

Un historiador puede pretender ser objetivo, pero esta objetividad nunca será inocente. La objetividad no es neutralidad ${ }^{6}$. La historia estudia el pasado para comprender el presente y construir el futuro. El presente, y el futuro (o la idea de futuro), condicionan siempre la mirada al pasado?

En el presente se construyen pues el pasado y el futuro, de modo que, como decía Benedetto Croce, y pusieron de manifiesto Bloch y Frebvre, desde los primeros Annales, toda historia es, en principio, historia contemporánea, ya que sólo desde el presente se escribe la historia y es el presente el que otorga sentido al estudio y al conocimiento del pasado ${ }^{8}$. El sentido de la historia debe ser, como ya decía Cascajero, su orientación hacia el cambio social, sin el cual, la Historia no tendría sentido más allá de la técnica, y de la pura acumulación de datos y el entretenimiento vacío?. No es posible pretender un futuro diferente sin la atención simultánea al pasado y al presente $^{10}$. Pero, si la Historia puede ser un instrumento para el cambio social, también puede ser un instrumento en manos del poder para lograr un cierto inmovilismo social. Aunque podamos aspirar a un mundo con menos diferencias sociales, hemos de tener en cuenta que, mientras las desigualdades existan, habrá beneficiarios de la desigualdad, muchos de los cuales no querrán ver su situación alterada. Es célebre la idea de 1984, de George Orwell, según la cual: "quien controla el pasado controla el futuro. Quien controla el presente controla el pasado" o, como puede aprenderse de la historia de Esaú según Lescek Kolakowski1" "un pequeño cambio del pasado puede reportar grandes beneficios".

Enric Marco Batlle afirmaba, en un emotivo discurso en recuerdo de las víctimas del nazismo en el Congreso de los Diputado, que "a los jóvenes, que no saben nada de la historia, les falta alguien que se la cuente", y quizás de él podamos extraer alguna enseñanza. Enric Marco, que era presidente de la asociación Amical de Mauthausen y otros campos, daba alrededor de 140 
conferencias anuales, y fue condecorado con la Cruz de San Jordi (la máxima distinción civil en Cataluña). Además de testigo, era historiador (cursó estudios de Historia en la Universidad Autónoma de Barcelona), e impostor, pues nunca fue deportado al campo nazi de Flossenbürg, ni a ningún otro ${ }^{12}$. Él mentía por que "así la gente me escuchaba más y mi trabajo divulgativo era más eficaz"13. Las verdades pueden ser subjetivas, pero las verdades existen, al igual que las mentiras, y aunque el historiador pueda tener una ideología política manifiesta, el fin nunca puede justificar los medios en las ciencias sociales. Desde nuestro punto de vista, a los jóvenes no sólo hay que contarles la historia, sino que también hay que inculcarles un espíritu crítico. Volveremos sobre ello más adelante.

$\mathrm{Al}$ igual que existen los impostores que manipulan la historia, también las instituciones pueden falsificar u ocultar ciertos datos, movidas por diferentes intereses (electoralistas, políticos, ideológicos, económicos...). En 2007, a escasos días de las elecciones autonómicas y municipales, la Junta de Castilla y León pagó 1,6 millones de euros por una espada Tizona que no era auténtica. La espada fue tasada en 200.000-300.000 euros por el Ministerio de Cultura. Se pagó pues 1,3 millones de euros por encima de este precio por una espada, conociendo la existencia de los informes que indicaban que no era la Tizona auténtica, para exponerla en la catedral de Burgos, eso sí, presentándola como la auténtica espada del Cid Campeador ${ }^{14}$. Más de un millón y medio de euros puede parecer mucho dinero por una espada, pero no se trata de una espada cualquiera, sino de la espada de un héroe regional, del que se puede transmitir una visión idealizada de un pasado legendario y glorioso que distorsiona la figura de Rodrigo Díaz de Vivar como personaje histórico, y de la época en la que éste vivió. Lo que se busca no es una mayor comprensión del pasado por parte de los ciudadanos, sino lo que Bermejo Barrera califica como "la ideología del patrimonio" o la "historia basura", que tiene como fin "contribuir a la trivialización de la cultura, y más concretamente de la Historia, la Arqueología y la Historia del Arte, con el fin de evitar que puedan convertirse en instrumentos de crítica social y política" ${ }^{\prime 5}$. Invertir en este tipo de reliquias supone invertir en el mal llamado turismo cultural ${ }^{16}$. Las instituciones, y sus ideólogos del patrimonio, se interesan, como dice Bermejo por "el turismo de masas, que se puede cuantificar, en función de cuyos grandes números se pueda medir el éxito económico, social y político de lo que ellos organizan, que es el sistema de exposiciones y mu- 
seos" ${ }^{17}$ cuya visita, sin una formación previa adecuada, tiene escaso valor, a menos que se pretendan convertir en "mecanismos de exhibición instrumentalizados políticamente, no con el fin de transmitir una ideología, sino por el contrario, de desideologizar a la sociedad" 18 .

El uso electoralista que los partidos políticos hacen de la historia quedó claramente de manifiesto en la polémica de los "papeles de Salamanca", en la que el gobierno del PSOE decidió proceder a la devolución de los documentos que le habían sido requisados a la Generalidad de Cataluña durante la Guerra Civil. Independientemente de que esta devolución fuese justificada o no, tema en el que no queremos entrar en este momento, el motivo de la devolución no fue científico sino completamente político (el apoyo de Esquerra Republicana de Catalunya).

Conocer el pasado posibilita comprender mejor el presente lo que permite adoptar una postura crítica hacia el mismo que contribuya a disminuir las desigualdades sociales. Como dice Plácido Suárez:

boy hay nuevas posibilidades de conocer el pasado, pero también nuevos trucos ideados por la clase dominante actuales para tergiversar el pasado, para bacer funcionar la memoria de un modo u otro ${ }^{19}$.

Desde nuestro punto de vista, las élites actuales condicionan los tres ámbitos que consideramos fundamentales en el trabajo de un historiador: la investigación, la divulgación, y la crítica.

Los investigadores son evaluados y deben obtener acreditaciones para poder promocionar en el mundo universitario. Como se dice en Programa VERIFICA Procedimiento para la Selección y el Nombramiento de las Comisiones de Evaluación para la Verificación de Títulos Universitarios Oficiales de la ANECA:

En coberencia con lo dispuesto en el artículo 25 del Real Decreto 1393/2007, las comisiones de evaluación estarán formadas por expertos del ámbito académico y, en su caso, profesional, del título correspondiente. Dichos expertos serán evaluadores inde- 
pendientes y de reconocido prestigio designados por $A N E C A^{20}$.

La ANECA (Agencia Nacional de Evaluación de la Calidad y Acreditación) es una agencia estatal pagada con fondos públicos y cuyos miembros son nombrados por el partido político gobernante, que tiene un papel importante a la hora de decidir quién puede juzgar la calidad del trabajo, de decir lo que es bueno y lo que es malo. Habría que señalar que su primer director fue un compañero de departamento de la entonces ministra Pilar del Castillo, cuando gobernaba el Partido Popular $^{21}$.

El condicionamiento a la investigación es muy claro, en primer lugar por que para investigar hace falta, entre otras cosas, tiempo y dinero. Las grandes investigaciones requieren un grupo de investigadores con un proyecto que debe ser financiado por una institución pública o privada que, lejos de ser neutral, tienen unos intereses políticos, ideológicos o económicos.

En el caso de los estudios históricos, artísticos, filológicos y arqueológicos se suele primar bien aquellos que pueden generar recursos turísticos (de nuevo la ideología del patrimonio y la historia basura), bien aquellos estudios regionalistas que permiten crear una identidad regional. Es lo que se denomina "áreas de interés prioritario", es decir, que son más importantes. Pero, ¿quién decide qué es más importante? No los especialistas, los científicos que al elegir un tema ya están considerando que es suficientemente importante, sino quienes gestionan los recursos, es decir, las instituciones que intervienen a la hora de decir qué se estudia, y qué no se estudia. Decíamos anteriormente que el historiador selecciona, desde el presente, el tema y los problemas que le interesan, pero esta selección está condicionada en gran medida por el poder.

La situación se agrava más si cabe en relación a la situación de los jóvenes investigadores, que para iniciarse en la carrera investigadora deben solicitar becas de investigación (predoctorales y posdoctorales), unas becas que son competitivas, pero en las que un joven investigador sólo en una parte relativamente reducida, pues su curriculum y expediente pesan menos, en el caso de los becarios predoctorales, que otros criterios como el curriculum del director de tesis, que el doctorado que realiza tenga una mención de calidad (algo que ajeno completamente a él), o que su proyecto de investigación sea de interés prioritario (no para él, que dedicará varios años a su estudio, sino 
para quien paga). Ello, unido a las malas condiciones de las becas, y las lamentables expectativas de futuro, condiciona sin duda la investigación.

Al igual que la investigación, la transmisión de los resultados de la investigación también está condicionada. Esta transmisión se hace fundamentalmente a través de exposiciones (realización de congresos, conferencias, ponencias, comunicaciones, etc.), ediciones (libros, revistas, artículos, etc.), y de la enseñanza. Los resultados científicos se transmiten, como dice Bermejo a través de:

libros, revistas y congresos que están controlados por editores, corporaciones y sociedades cientificas, que pueden funcionar como elementos de censura, en tanto que siempre tratarán de defender, en el caso de las ciencias, el paradigma existente y los intereses de poder científico, económico y militar que boy día se esconden tras la realización de la investigación científico-técnica².

La organización y la asistencia a congresos requiere el amparo económico de las instituciones, bien directamente, bien por la financiación por parte de los proyectos de investigación. La solicitud y justificación de los gastos conlleva una pesada carga administrativa que dificulta de manera considerable el trabajo del investigador, un trabajo cada vez más burocratizado.

Las obras editadas están condicionadas por las evaluaciones de la calidad, y los índices de impacto. Los investigadores deben obtener primero becas o contratos (predoctorales y posdoctorales), y luego pasar una serie de acreditaciones que obligan a publicar, y no en los sitios que quiera el investigador sino en aquellos en los que algunos expertos han decidido, interesada o desinteresadamente, que es más importante publicar. A un investigador le es académicamente más beneficioso publicar un mal artículo en una revista bien posicionada que un buen artículo en una revista que esté mal posicionada (por muy alta que sea la calidad de ésta según otros parámetros diferentes a los de los evaluadores). Uno puede publicar (hacer público) lo que quiera, pero debe editarlo donde alguien ha decidido que es más valioso para la ciencia.

Bermejo Barrera considera que puede definirse la evaluación científica: 
como un proceso social y politico de captación de dinero y de control de las instituciones que desarrollan las comunidades cientificas. Ese proceso es inevitable, pero está viciado porque en él las comunidades dominantes, por razones económicas o politicas, imponen sus criterios de evaluación para dejar marginadas a las demás y copar el campo académico e investigador ${ }^{23}$.

La medición de la calidad de la investigación, lejos de ser neutra, favorece a unos investigadores frente a otros, y a unas áreas científicas frente a otras en la obtención de los recursos y de cuotas de poder y favorece también a unas lenguas sobre otras, siendo la inglesa la gran beneficiada de este proceso.

Calidad no es cantidad, se supone. Sin embargo, se mide la calidad por la cantidad de citas que los artículos publicados reciben. Es cuestionable el que la unidad de medida del conocimiento sean los artículos, y que la calidad de estos pueda medirse por las citas recibidas. En primer lugar, de este modo se quita valor a algo tan importante, especialmente en las disciplinas humanísticas, como los libros. Por otro lado, la calidad de un artículo se mide, no por lo que aporta al conocimiento, sino por la cantidad de citas recibidas, obviándose que en los campos de las ciencias sociales y jurídicas y en los de humanidades, muchas citas suelen ser negativas ${ }^{24}$, o deberían serlo si se lleva a cabo la función crítica de los científicos. Este valor que se le da a las citas como medida de la calidad lleva a citar a amigos y silenciar a enemigos y por supuesto a la autocita irrelevante ${ }^{25}$. Del mismo modo, los evaluadores de la calidad favorecen a unas revistas frente a otras con criterios que pueden ser puestos en cuestión, como por ejemplo el de que una revista rechace el 40\% de los artículos recibidos, hecho que podría indicar que esta recibe muchos artículos de baja calidad (por que de lo contrario hemos de suponer que serían aceptados, o que se rechazan artículos que no deberían rechazarse), y sin embargo es un criterio de calidad de la FECYT ${ }^{26}$, como lo es el estrictamente económico según el cual es aconsejable que el porcentaje de ejemplares vendidos por suscripción o por venta directa sea superior al 40\% de la tirada ${ }^{27}$, o el criterio según el cual el porcentaje de trabajos financiados por organismos públicos o privados de investigación sea mayor del $40 \% 0^{28}$, como si el origen del dinero con el que se hacen los artículos le diese calidad a estos. Desde nuestro punto de vista, si hubiese que poner un criterio económico de calidad, este sería el no cobrar a los autores por publicar, práctica habitual 
entre algunas de las más prestigiosas publicaciones científicas. Un criterio muy cuestionable era el que restaba valor a las publicaciones realizadas en la editorial de la propia universidad. Este criterio ponía en peligro los intereses de las editoriales universitarias que no tardaron en movilizarse y conseguir que el gobierno eliminase este criterio que consideraban discriminatorio ${ }^{29}$.

Por otra parte, el sistema de evaluación por pares ciegos tiene grandes carencias al basarse en el trabajo de investigadores anónimos, no remunerados, a los que no se les reconoce su esfuerzo, y que no siempre pertenecen al área del autor al que están evaluando, y al que presuntamente no conocen. Por supuesto surge la pregunta ¿quién evalúa a los evaluadores? Un criterio de calidad de la FECYT es precisamente que se controle la calidad de los informes de evaluación ${ }^{30}$, pero quien tenga un contacto más o menos habitual con los informes habrá podido comprobar que hay informes cuya calidad deja mucho que desear, quizás por que los informantes no reciben ningún tipo de formación al respecto, o por que los criterios que emplean son excesivamente subjetivos, lo que es patente especialmente cuando el informe es realizado por un investigador de un área de conocimiento diferente a la del autor del artículo.

$\mathrm{Al}$ aplicarse en áreas de humanidades y ciencias sociales criterios de otras áreas científicas se llega a incongruencias como el valorar las citas recibidas sólo en los primeros dos años de vida del artículo, y en ciertas revistas. Teniendo en cuenta que la revista debe ser distribuida, el artículo que cita redactado, enviado, revisado por pares ciegos (y por un tercer revisor en caso de duda), corregido, y publicado tras varias pruebas de imprenta, se hace difícil poder publicar en poco tiempo un artículo que cite otros del año anterior. A esto hay que añadir que muchas revistas anuales son editadas en diciembre y distribuidas en enero del año siguiente, dificultando más el que sus artículos sean citados en dos años, lo que hace que sea recomendable, de cara a las evaluaciones, editar las revistas en enero. Sea como sea, no valorar positivamente aquellos artículos que siguen siendo importantes para la ciencia después de varios años parece una incongruencia.

Algo que tampoco suele cuestionarse es el hecho de que la vida media de un artículo es de cinco años, pidiéndoseles a los científicos que en sus $\mathrm{CM}$ rricula limiten sus publicaciones y actividades a los últimos cinco años ${ }^{31}$. Esto es algo que tiene poco sentido en las áreas de humanidades y ciencias sociales, 
donde un artículo, sobre todo si tiene la calidad que debería buscarse, puede seguir estando de actualidad después de un tiempo muy superior. Como dice Bermejo:

Podríamos pensar que pretender aplicarles a todos el mismo patrón es fruto de la ignorancia. Sin embargo, dado que los académicos e investigadores no son, por definición, ignorantes, lo que es en realidad un intento de privilegiar unos tipos de curricula sobre otros en el marco de la lucha académica para lograr la consecución de fondos y el control de las instituciones ${ }^{32}$.

Por otra parte

el número de citas en el sistema ISI pierde sentido si comparamos las medias entre diferentes campos. Entre los 400 casos analizados por Goodall ${ }^{33}$ de grandes investigadores que ocupan cargos de prestigio, tenemos una oscilación de citas por años entre 1.095, en el caso de la medicina clinica, y 1.234, en el caso de la biología molecular (serían los dos autores de mayor cota), a 35 en el campo de las humanidades y 84 en el campo de las ciencias de la computación ${ }^{34}$.

Como efectivamente, los académicos no son tontos, recurren a estrategias de promoción y autopromoción que explican hechos como el autoplagio o la republicación de sus mismos artículos con otros títulos diferentes.

Otra forma de transmisión de los conocimientos es la que se realiza a través de la enseñanza, sin la que el estudio del pasado perdería gran parte de su sentido e implicación social. En el caso de la Historia y de las ciencias afines, se plantean varios problemas en este aspecto. En la enseñanza universitaria la calidad de la docencia tiene un peso mucho menor en la promoción de los profesores que su trayectoria investigadora. De hecho, a diferencia de lo que sucede en el profesorado de enseñanzas medias, que deben cursar un master en el que presuntamente se les debería enseñar a enseñar, los profesores universitarios no necesitan ninguna formación didáctica específica.

Los planes de estudio universitarios tienen carencias graves que no parece que vaya a solucionar el Proceso de Bolonia, que ha sido utilizado de excusa para tratar de eliminar varias carreras humanísticas por el único criterio altamente cuestionable de no ser rentables económicamente, o para eli- 
minar asignaturas (cuatro por área en el Grado de Historia de la Universidad de Salamanca), pudiendo de esta manera amortizar plazas, sacando así de la carrera universitaria a muchos jóvenes investigadores que pasan a ser innecsarios para el sistema académico universitario. Pero, pese a todas las carencias del sistema universitario, donde de verdad se ha producido un rotundo fracaso en el sistema educativo ha sido en los grados medios, especialmente en la enseñanza obligatoria.

El que ésta, la educación obligatoria, esta muy politizada es algo que salta a la vista al prestar atención a las leyes de educación, y a cómo cada partido que llega al poder trata de imponer, que no consensuar, su modelo. Esta es una de las causas determinantes del panorama educativo actual, un panorama desolador que algunos no quieren ver o no quieren que se vea, y que está controlado por quienes tienen el poder en temas educativos, a los que algunos han denominado "la secta pedagógica" o la "pedagocracia" 35 .

Los profesores de las enseñanzas medias, en la educación pública, son funcionarios que deben realizar una oposición en cada comunidad autónoma. En estas oposiciones, por encima de las capacidades del opositor se valoran más otros hechos como el tiempo que el opositor lleva fracasando para conseguir una plaza, o los cursos de "formación" por los que ha pagado. Es mejor haber hecho 4 malos exámenes de oposición que haber hecho un examen de 10, o que tener dos carreras y un doctorado. Esto es responsabilidad directa de quienes ostentan el poder en materia educativa, en este caso las comunidades autónomas y los sindicatos que presionan para adaptar los exámenes a sus propios intereses. De esto modo, para reducir las listas de interinos, en los últimos años se viene realizado un examen mucho más fácil que en años anteriores (se ha eliminado la parte práctica del examen, y se extraen 5 temas a elegir uno, en lugar de 2 a elegir 1 como se hacía anteriormente), pero en las siguientes oposiciones se volverá a subir la dificultad del examen, además de exigir un master universitario y un certificado oficial de un segundo idioma, todo ello con el fin, no de mejorar la calidad de los docentes, sino de reducir las listas de opositores.

Si en el caso de asignaturas como matemáticas podemos considerar una imprudencia dar preferencia a un profesor mediocre sobre uno brillante pero más joven, en el caso de Geografía e Historia surge el peligro adicional de que se politice y regionalice la enseñanza de la Geografía, la Historia y la 
Historia del Arte. Por poner un ejemplo, en el examen de oposición para profesor de enseñanza secundaria de Extremadura del año 2004 la parte práctica de $\mathrm{H}^{\mathrm{a}}$ del Arte consistió en un análisis de una obra de Godofredo Ortega Muñoz. Sin querer desvalorizar la obra del pintor pacense, no se trata, a nuestro parecer, de un autor en absoluto fundamental para la comprensión del arte contemporáneo y menos en un nivel de enseñanza secundaria. Si conocemos el hecho de que Sindicato de Trabajadores y Trabajadoras de la Enseñanza de Extremadura (STE-EX), está ubicado en la calle Godofredo Ortega Muñoz ${ }^{36}$ de Badajoz, podremos entender mejor las caras de satisfacción de algunos aspirantes a profesores que contrastaba con la cara de desconcierto de otros. En este ejemplo, podrían darse muchos otros, aprobar el examen era más fácil para los aspirantes extremeños.

Una gran herramienta de control ideológico pueden ser los libros de texto. Mientras que en la universidad se renuevan poco los manuales, por que no son muy rentables ni económica ni académicamente (el esfuerzo de escribir un libro es menos rentable para la promoción profesional que el de publicar artículos), los libros de texto de secundaria, que no son gratuitos ni baratos pese a que se trate de una educación obligatoria, sí son muy rentables para las editoriales, precisamente por su carácter obligatorio. Si la evolución de la ciencia no es tan grande como para hacer necesario renovar constantemente los manuales universitarios, cuesta comprender, al margen de intereses políticos y económicos, la necesidad de cambiar cada pocos años todos los libros de texto en la enseñanza secundaria.

Por otra parte, cabe preguntarse si debemos utilizar la enseñanza de la historia para crear identidades regionalistas con fines políticos. Sobre la manipulación educativa ya alertó la Real Academia de la Historia en 2000 y poco se ha cambiado desde entonces. El mismo tema en un libro de la misma asignatura y la misma editorial puede cambiar mucho dependiendo de la comunidad autónoma ${ }^{37}$.

Como decíamos, la formación de una actitud crítica debería ser una función de los historiadores, y en general de todo universitario. Esta crítica debería favorecer el intercambio de ideas y dificultar la manipulación del pasado que realizan quienes están en el poder o de los impostores. Es la crítica la que permite destapar engaños como los de Enric Marco Batlle, Iruña Veleia, o la cueva de Zubialde, y la que posibilita que no todo el mundo aplauda 
o quede indiferente cuando un ex-presidente del gobierno afirme, en una conferencia universitaria, que el problema de España con Al Qaeda y el terrorismo islámico empezó en el siglo octavo, cuando España "recientemente invadida por los moros, rehusó a convertirse en otra pieza más del mundo islámico y comenzó una larga batalla para recobrar su identidad"38. La creación de identidades es precisamente uno de los usos, o abusos, que se hace de la historia, y los historiadores deben desempeñar un papel activo denunciando los abusos, las falsedades, las falsificaciones y a los falsarios ${ }^{39}$.

Sin embargo, la politización de la ciencia hace que en no pocas ocasiones la crítica sea silenciada u ocultada por temor quizás a las consecuencias. Los pares ciegos que evalúan los artículos y libros, al igual que los evaluadores de proyectos, deben ser anónimos para evitar represalias, las reseñas de libros pocas veces son críticas, los trabajos de grado y las tesis doctorales en raras ocasiones obtienen una nota inferior a sobresaliente cum laude, lo que crea la ilusión de que todas las tesis tienen la misma calidad. Las críticas y debates en congresos, seminarios o conferencias son tan escasos como necesarios.

Es de sobra conocido que los historiadores no pueden predecir el futuro, pero sí actuar para construirlo. Para ello deben trabajar por comprender el presente, y convertir el conocimiento del pasado en una herramienta para la igualdad ${ }^{40}$. En este artículo hemos tratado de invitar a la reflexión y al debate sobre algunos de los mecanismos que utilizan las diferentes oligarquías para mantener el poder. Como decía Cascajero, "mientras haya desigualdad en el mundo, habrá beneficiarios de la desigualdad" ". En este sentido los historiadores tenemos una gran responsabilidad con la sociedad, debemos ser, no sólo investigadores del pasado, sino hombres de acción en el presente.

El futuro del pasado es impredecible, pero sí podemos pronosticar que será construido y divulgado por los hoy jóvenes investigadores. La mayoría de los becarios predoctorales serán dramáticamente apartados de la carrera universitaria después de muchos sacrificios personales, pero también es cierto que la mayoría de los profesores universitarios del futuro habrán sido antes becarios predoctorales. Aunque la Universidad de Salamanca presta poca atención al personal investigador en formación, negándoles incluso el derecho a votar democráticamente a su Rector por motivos económicos ${ }^{42}$, debería ser consciente de que son precisamente los jóvenes investigadores quienes deberán construir el futuro de la universidad más vieja de España. Y ellos, 
en el futuro, deberán elegir si quieren mantener, o transformar, los mismos mecanismos de poder existentes actualmente.

\section{NOTAS}

*Personal investigador en formación (FPI) del MICINN, adscrito al proyecto HAR200913597.

${ }^{1} \Delta \mathrm{x} \cdot \Delta \mathrm{p} \geq \hbar / 2$.

${ }^{2}$ Cascajero, Juan, “Tiempo y tiempos para la Historia Antigua”, en Gerión, no 18 , 2000, pp. 17-41, esp. p. 19, nota 4.

${ }^{3}$ Plácido SuÁrez, Domingo, "La historia entre los tiempos", Gerión, vol. extra, 2007, pp. 7-23, esp. p. 9.

${ }^{4}$ PláCIDO SuÁreZ, Domingo, La historia entre los tiempos..., p. 14.

${ }^{5}$ CASCAjero, Juan, Tiempo y tiempos..., p. 37.

${ }^{6}$ Bermejo BARrerA, José Carlos, Sobre la Historia considerada como poesía, Akal, Madrid, 2005, p. 14.

${ }^{7}$ CASCAjero, Juan, Tiempo y tiempos..., p. 39.

${ }^{8}$ Bermejo Barrera, José Carlos, Sobre la Historia..., p. 31

${ }^{9}$ CASCAjero, Juan, Tiempo y tiempos..., p. 18. Todo historiador, y todo humanista, debería preguntarse para qué sirve su trabajo; al respecto véase: Sobre la utilidad de las humanidades véase el sugerente artículo de WELZER, Harlad, “ßBasta ya de inútiles!”, Revista de Occidente, $\mathrm{n}^{\circ} 313,2007$, pp. 5-12, y la contestación de SEEL, Martin, "La paradoja de la utilidad: sobre la legitimidad de las ciencias humanas", ibidem, pp. 13-16.

${ }^{10}$ Cascajero, Juan, Tiempo y tiempos..., p. 20.

${ }^{11}$ KOLAKOWSKI, Lescek, La clave celeste, Melusina, Barcelona, 2006, pp. 34-38.

${ }^{12}$ Véase al respecto el dossier "El caso Enric Marco" del Centro de Investigaciones sobre los Republicanos Españoles en los Campos Nazis, en

$<$ http://www.exilioydeportacion.com/newmarco.htm> (última consulta 11-I-2010). Sobre el caso existe un reciente documental: Ich bin Enric Marco (2009), dirigido y guionizador por Santiago Fillol y Lucas Vermal.

${ }^{13}$ Diario 20 Minutos, 11 de mayo de 2005. Se trata de una noticia de agencia que aparece en otros medios. <http://www.20minutos.es/noticia/22947/0/Mauthausen/deportados/espanoles $>$ (última consulta 11-I-2010).

${ }^{14}$ Diario 20 Minutos, 25 de mayo de 2007. Se trata de una noticia de agencia que aparece en otros medios. <http://www.20minutos.es/noticia/239077/0/tizona/es/falsa/> (última consulta 11-I-2010).

${ }^{15}$ BERMEJO BARRERA, José Carlos, "La ideología del patrimonio y el nacimiento de la historia basura”, Gallaecia, no 25, 2006, pp. 289-304, esp. p. 292.

${ }^{16}$ Véase: SENABRE LÓPEZ, David, “¿Es cultura el "turismo cultural”?, en Foro de Educación, $\mathrm{n}^{\mathrm{o}} 9$, pp. 71-79.

${ }^{17}$ BERMEjO BARRERA, José Carlos, La ideología del patrimonio..., p. 296.

${ }^{18}$ Idem. 
${ }^{19}$ PLÁCIDO SUÁREZ, Domingo, La historia entre los tiempos..., p. 17.

${ }^{20}<$ http://www.aneca.es/media/164600/verifica_comites_v04_081126.pdf>(última consulta 19-I-2010).

${ }^{21}$ BERMEJO BARRERA, José Carlos, “«Words, orly words». El proceso de Bolonia o cómo cuadricular el caos”, en Ingeniería y territorio, 87, 2009, pp. 14-19, esp. p. 16.

${ }^{22}$ Bermejo Barrera, José Carlos, Sobre la Historia..., p. 51.

${ }^{23}$ BERMEJO BARRERA, José Carlos, "La inconsistencia de las evaluaciones científicas: elogio del silencio”, en RIFP, n² 27, 2006, pp.168-185, esp. p. 178.

${ }^{24}$ BERMEJO BARRERA, José Carlos, La inconsistencia de las evaluaciones científicas..., p. 179.

${ }^{25}$ Por ejemplo, mi artículo PÉREZ MIRANDA, Iván, “Género y análisis de videojuegos”, en Hernández Huerta, José Luis; SÁnChez Blanco, Laura; PÉREZ MirandA, Iván, Temas y perspectivas sobre educación. La infancia ayer y hoy, Globalia/AJITHE, Salamanca, 2009, pp. 313328 , no tiene ninguna relación con el tema aquí tratado, pero acabo de citarlo.

${ }^{26}$ LÓPEZ-CÓZAR, Emilio Delegado; RUIZ-PÉREZ, Rafael; Jiménez-Contreras, Evaristo: La Edición de Revistas Cientificas: Directrices, Criterios y Modelos de Evaluación, Granada, 2006, p. 78, en <http://recyt.fecyt.es/documentos/Fecyt.pdf> (última consulta 11-I-2010).

${ }^{27}$ LÓPEZ-CÓZAR, Emilio Delegado; RuIZ-PÉREZ, Rafael; Jiménez-Contreras, Evaristo: La Edición..., p. 24.

${ }^{28}$ Ídem.

${ }^{29}$ Véase al respecto el BOE, no 289, 1-XII-2009, Sec. III, disponible electrónicamente en: <http://www.boe.es/boe/dias/2009/12/01/pdfs/BOE-A-2009-19218.pdf>; véase también:

$<$ http: / $/$ www.eusal.es $/$ index.php?option $=$ com_content\&view $=$ article\&id $=311 \% 3$ Alas-editoriales-universitarias-dejan-de-estar-discriminadas-en-la-evaluacion-delprofesorado\&catid $=126 \% 3$ Aactualidad $\&$ Itemid $=114 \&$ lang $=e s>\quad$ (última consulta 19-I-2010).

${ }^{30}$ LÓPEZ-CÓZAR, Emilio Delegado; RuIZ-PÉREZ, Rafael; Jiménez-Contreras, Evaristo: La Edición..., p. 23.

${ }^{31}$ BERMEJO BARRERA, José Carlos, La inconsistencia de las evaluaciones científicas..., p. 179.

${ }^{32}$ BERMEJO BARRERA, José Carlos, La inconsistencia de las evaluaciones cientificas..., p. 181.

${ }^{33}$ GOODALL, A. H., Socrates in the Boardroom. Why Research Universities Should be Led by Top Scholars, Princeton University Press, 2010, pp.141

${ }^{34}$ BERMEJO BARRERA, José Carlos, “¿Quién debe gobernar las universidades? Ensayo sobre la legitimidad académica" en <http:// firgoa.usc.es/drupal/node/45096> (última consulta 19-I-2010).

${ }^{35}$ Véase: "Manifiesto Pedagógico: No es verdad", en:

$<$ http://www.redires.net/NO_ES_VERDAD.pdf> escrito en respuesta a MORENO CASTILLO, Ricardo, Panfleto antipedagógico, Editorial leqtor, Barcelona 2006, y contestado por este mismo autor: MORENO CASTILlO, Ricardo, "No es verdad que no sea verdad", disponible en <http:/ /amesweb.tripod.com/noesverdadquenoseraverdad.pdf $>$ (última consulta 11-I2010); RuIZ PAZ, Mercedes, La Secta Pedagógica, Unisón, Madrid, 2003; BERnaBÉ, Maximiliano, "Contra la pedagocracia", en http:/ / www.elinconformistadigital.com/modules.php?op $=$ modload\&name $=$ News\&file $=$ ar- 
ticle\&sid $=1744$

JOVÉ MASANA, Xavier, "Contra la pedagocracia”, en:

<http://www.elpais.com/articulo/opinion/pedagocracia/elpepiopi/20080105elpepiopi_8/Tes/> (última consulta 11-I-2010).

${ }^{36}$ Concretamente en C/ Godofredo Ortega Muñoz 34A Entrepl. Oficina 1. CP: 06011 BADAJOZ.

${ }^{37}$ En 2007 Manuel Romero elaboró para el periódico El Mundo un análisis comparativo sobre los libros de texto, titulado "El escándalo silencioso que va minando España", que pone de manifiesto los graves problemas que crea la descentralización educativa. El análisis puede consultarse electrónicamente en:

$<$ http:/ / www.tolerancia.org/asp/index2.asp?area $=$ area6\&p $=273 \& \mathrm{t}=6>$.

$<$ http:/ $/$ www.tolerancia.org/asp/index2.asp?area $=$ area6\&p $=273 \& t=9>$.

$<\mathrm{http}: / /$ www.tolerancia.org $/$ asp $/$ index2.asp?area $=$ area6\&p $=274 \& \mathrm{t}=5>$.

$<$ http://www.tolerancia.org/asp/index2.asp?area $=$ area6\&p $=275 \& \mathrm{t}=1>$.

$<$ http://www.tolerancia.org/asp/index2.asp?area $=$ area6\&p $=275 \& \mathrm{t}=7>$.

$<$ http://www.tolerancia.org/asp/index2.asp?area $=$ area6\&p $=275 \& \mathrm{t}=9>$.

$<$ http:/ $/$ www.tolerancia.org/asp/index2.asp?area $=$ area6\&p $=276 \& \mathrm{t}=1>$.

En Castilla y León las asociaciones Plataforma Regional Pro-Identidad Leonesa y Promonumenta, realizaron un análisis de los contenidos de los libros de texto de la Comunidad Autónoma de Castilla y León con base a la cual se remitió una queja al Procurador del Común. Véase: <http://reinolvidado.blogspot.com/2009/02/la-manipulacion-en-lasasignaturas-de.html> (última consulta 11-I-2010).

${ }^{38}$ La lección de José María Aznar impartida en septiembre de 2004 en la universidad de Georgetown puede consultarse electrónicamente en:

<http://www.elsiglodeuropa.es/siglo/historico/politica/politica2004/618Aznar.htm> (última consulta 19-I-2010).

39 "Falso, falsario, falsificación, falsedad" fue el título del XI Coloquio Internacional de ARYS, celebrado en diciembre de 2006, y cuyos resultados se publicarán en breve en la revista $A R Y S$.

40 "El conocimiento del pasado: una herramienta para la igualdad" es precisamente el título de una serie de seminarios sobre historia de género realizados en la Universidad de Salamanca, algunas de cuyas intervenciones fueron publicadas en SEvillano SAn José, Carmen; Rodríguez Cortés, Juana; Olarte Martínez, Matilde; LAHOZ, Lucía (Eds), El conocimiento del pasado, una herramienta para la igualdad, Plaza Universitaria Ediciones, Salamanca, 2005.

${ }^{41}$ Cascajero, Juan, Tiempo y tiempos..., p. 40, nota 47.

${ }^{42} \mathrm{Se}$ pretende que el personal investigador contratado en formación pague una cuota anual por realizar el trabajo por el cual son contratados, pagando esta cuota un investigador podría votar como un estudiante de tercer ciclo, pues en los estatutos "no se prevé la participación del personal investigador en formación, o con la terminología normativa del 2003, de los becarios", como se dice en la "Resolución de la Junta Electoral de la Universidad de 23 de octubre sobre reclamacio- 
nes al censo provisional para la elección de rector", disponible electrónicamente en <http://www.usal.es/webusal/files/Resolucion_contestacion_reclamaciones_censo.pdf > (última consulta 19-I-2010). 